\title{
A Review of Smart Grids and Their Future Challenges
}

\author{
Wen $\operatorname{Tian}^{1}$ \\ ${ }^{1}$ Electrical Engineering and the Automatization Specialty, Inner Mongolia University of Technology, Ming An \\ Tu Street, Xilinhot, China
}

\begin{abstract}
With the development of science and technology and the increasing emphasis on renewable energy, smart grids are being vigorously developed in many countries and regions including Europe, the United States and China. The smart grid is the main practice trend of efficient transmission of modern power resources. It has the advantages of good power transmission, low resource loss and strong correlation of resources transmission. Therefore, it occupies an important position in large-scale utilization of power resources. Thus, this paper, based on the theory of smart grid, discusses the research direction, practical innovation and application of smart grid, provides theoretical reference for the comprehensive utilization of power resources in China, and promotes the intelligent exploration of domestic electric resource application.
\end{abstract}

\section{INTRODUCTION}

Electricity supply is the main source of power for social development. With the cyclical adjustment of the domestic economic structure, the proportion of the loss of social resources has also gradually increased. The research and application of renewable energy have gradually become the trend of developing energy policies in large countries. Among many renewable energies, electric power has been the research focus due to its wide application and strong renewable ability. Therefore, the development of power systems for power generation, substation, transmission and storage has been enhanced, where smart grid has become the top priority. In order to give full play to the role of power supply in social innovation, it is necessary to figure out the source of power supply, but also to constantly construct new applications and management modes of power resources. The smart grid is based on the power transmission mode born in this background, with the characteristics of intelligent, low loss, high transmission and plays a fundamental role in the modern power transmission. Research on smart grid is a multi-disciplinary and multidomain topic, and this topic also has countless research value. This article will address the ultimate challenges for smart grids in the future. The next part, first of all, will be a brief introduction of smart grid, followed by the discussion of its research direction and the challenges faced, and its application part in final part.

\section{SMART GRID INTRODUCTION}

\subsection{The General Structure}

Smart grid is the intellectualization of power grid. It is built on the basis of integrated and high-speed two-way network communication structure. Through advanced power transmission framework, a more structured and hierarchical power supply mode is formed [1]. Smart grids divide the integrated structure of traditional grid supply into multiple control sections. Compared with the traditional structure, smart grid can meet the demand of large scale electricity market in twenty-first Century in terms of self regulation, user information management and defense against attacks.

\subsection{Transfer Method}

Combined with the basic application structure of the current domestic smart grid, we divide the smart grid into three parts: smart substations, smart substations, smart meters, intelligent switching terminals, intelligent dispatching and smart appliances. The power resource analyzes the effect of power transmission through the technology of electric power sensing and detection, and applies intelligent terminal control, device monitoring and other technical transmission parts. All the power transmission parts are integrated together to complete the terminal transmission of power grid information [2].

\section{$2.3 \quad$ Features}

Based on the traditional power transmission structure, the smart grid uses digital program control to replace manual operation to make the power transmission structure of the power grid structure stronger and to increase the power supply fluency. Therefore, the smart grid has the characteristics of intelligence and integration. At the same time, the total amount of transmission of smart grid resources is automatically adjusted according to the demand of regional power supply and the effective connection between intelligent focuses can avoid the

* Corresponding author: yeah.net.tw@163.com 
power loss in the transmission of power grid and effectively solve the problem of uncoordinated regional power supply problem, increasing the security and adjustability of the grid supply.

\section{RESEARCH DIRECTION}

\subsection{Stability}

The stability of practical application and the reliability of power transmission in smart grid account for more than $50 \%$ of smart grid in recent years, which is the main aspect of smart grid exploration. Some domestic scholars pointed out: the operation of smart grid relies mainly on the identification and docking of digitized program codes. As long as we set the program codes in accordance with the actual situation of regional power transmission, there is no problem of fluctuation in power transmission. This proves that the reliability of smart grids is high enough; At the same time, some scholars also believe that the program operation and overall structure operation of the smart grid can only be regarded as a branch of the development of digital digitization programs, and can not be used as a guarantee for comprehensive transmission of power grids. Therefore, we should make the smart grid power transmission unstable problem coping plan.

\subsection{Renewable Energy Utilization}

The integrated operation of smart grid power transmission mode also needs to take into account the role of smart grid in the loss of social resources. The development of traditional power resources mainly adopts the way of coal combustion to supply resources. In order to realize the function of electric power in the development of green society, we should make use of the advantages of smart grid to realize the reverse research of smart grid technology and combine it with new energy sources such as hydropower, wind energy, solar energy and tidal energy, gradually forming the new resource application mode of smart grid generation, supply and application, and do a comprehensive exploration of modern resource structure. This is also the main aspect of the current application and future development of smart grid in China.

\subsection{Demand-Side Design}

The design of the demand side of the smart grid is also the main aspect of the supply of multiple power resources in the future. In the analysis of modern power transmission structure, it occupies $30.11 \%$ of the proportion. Among them, $15.19 \%$ of the researchers believe that the current smart grid terminal detection technology, market demand analysis technology, are from the perspective of the development needs of the smart grid level, yet the future of smart grid innovation in the depth of exploration is not enough, which is the core point of smart grid long-term exploration; The remaining $14.92 \%$ of the scholars believe that the design of the terminal demand of smart grid has been adapted to the large-scale development needs of society, future needs of smart grid demand side design should be further explored in terms of terminal services, security protection, capacitor transmission quality.

\subsection{Power Storage Equipment}

Power storage equipment is the main component of practical application of smart grid, and it is also the basic power supply that can not be ignored in the supply of modern society resources. The development of power storage equipment in China's smart grid is accompanied by the development of smart grid. Therefore, comprehensive analysis of the power supply model can just be protecting the existing state of the smart grid power storage equipment. Common smart grid power savings models include lithium batteries, new lithiumsulfur batteries, etc. These transmission channels are all transmitted through the power structure, implementing a cyclical cycle of power supply, better power loss, durability of power supply.

\subsection{Power Transmission}

The most direct representation of smart grid in the transmission processes are the smart substation, smart distribution network, and intelligent scheduling [3]. On the one hand, the smart transmission structure of the existing smart grid in China has the characteristics of key monitoring and line tracking analysis. For example, area $\mathrm{A}$ is divided into three buses and 300 sub-lines. In the monitoring process of the transmission line of the smart grid, the bus sections in the transmission lines are mainly monitored while the terminal is installed on 300 sub-lines monitoring point, so that the entire power transmission process of the smart grid are subject to monitoring and management; On the other hand, in the analysis of transmission lines in smart grid, the terminal monitoring and line analysis are interrelated. In the actual application process, a certain extension space is reserved for the regional power transmission, so as to provide guidance for the transmission and supply of power resources in the later stage.

\section{CHALLENGES}

Smart grid plays an increasingly important role in the power supply in China. Combined with the analysis of the power supply structure of domestic smart grid, smart grid applications in China have implemented comprehensive exploration of the power supply and utilization of resources of the scientific mode of regulation, but the development of smart grid is still in the initial stage, the development of smart grid there are still many challenges:

On the one hand, there are some problems in the smart grid's resource supply and structural coordination processing, such as poor technology processing effect 
and poor availability of resources, which are the internal problems in the practical application of smart grid.

On the other hand, in the smart grid, there is a gap between the resource application structure and the market demand, the actual transmission structure is insufficient and the power for future development is limited, which are also factors that restrict the further development of the smart grid.

In addition, the development of China's smart grid has shifted from the initial stage to the comprehensive exploration. The development of the smart grid system in the transitional phase requires more specialized personnel and a more adequate financial base. At present, the development of smart grid in China is relatively tight and its development structure is slow. It is one of the challenging factors blocking the future development of smart grid.

\section{DISCUSSION AND APPLICATION}

To promote the comprehensive analysis of smart grid and its future challenges, it is necessary to combine the research core of the current smart grid and the future research area to build a more hierarchical and phased development trend of smart grid.

\subsection{Application of High Energy Testing System}

Compared with the traditional power transmission structure, the smart grid has more comprehensive power transmission and higher controllability in all parts of the power supply. First of all, the application of high performance detection system in smart grid shows as smart terminal device and smart grid transmission system. These systems utilize a digital program structure that sets the data collection hub for current transfer at all stages of the smart grid. When the user applies the current, the smart grid monitoring system will be quickly related to the current transmission statistics; Second, the application of high-performance smart grid monitoring system has achieved the data resource collection of twoway power transmission, and formed a comprehensive analysis of power transmission as the leading regional power resources relying on electricity transmission monitoring program. While the modern power supply structure is operated periodically, as long as the end users carry on the power supply, the detection system will have a terminal data control. Such a regular power transmission operation can avoid large-scale obstacles generated by integrated transmission of intelligent terminal control system, and plays an indispensable role in modern resource transmission.

\subsection{High Power Distribution System}

Based on the existing self healing system, smart grid further expands the intelligent terminal control structure, thus forming the current transmission structure of power distribution system and fault monitoring operation synchronously.
First, the current smart grid control structure uses program language information management to distribute the relevant procedures inside the grid power transmission, are distributed in the main transmission network branches so that when there is a line failure in the transmission part of the smart grid, the power transmission in the smart grid tends to adjust naturally and the mode of operation of the structure gradually forms a mode and predictable power transmission structure.

Second, the smart grid adopts bidirectional current transmission to realize phased management of power transmission structure. For example, there is a big difference in the monitoring of the power transmission in the sub-line part and the resource monitoring in the bus line part. The operation of the current transmission mode needs a more comprehensive information provision guarantee. If the intelligent power transmission network monitors the transmission line for any problems, the overall structure of the power transmission may also undergo a large-scale adjustment to ensure the security of the power supply of the power line.

\subsection{Advanced Management AAML Model}

In order to further play the role of smart grid in social development, in addition to the technical needs of smart grid supply and transmission, the management mode of smart grid should be further analyzed. The AAML model is based on the structural requirements of the macro-processing of the national power grid and carries out the smart grid user layer, the power grid business processing layer, the application service layer, and the system layer [4].

When the terminal information appears on the power transmission line terminal, the AAML mode will further process the power transmission in the smart grid according to the mode operation. When there is a shortage in line transmission, AAML will automatically transmit every cycle of the power grid to adjust and guide the mode of power transmission, and fully adapt to the needs of modern power supply. The periodic processing of modern power supply structure is regulated according to the demand of current transmission in different directions, until it constructs a controllable resource application guarantee.

\subsection{Effective Analysis of Smart Grid External Market}

Combined with the actual demand for resource transmission in modern systems, the planning and development of smart grid in the future needs to be analyzed from the aspects of economic input and personnel input to ensure the regionalized application to adapt to the overall power supply. For example: smart grid program update phase, the application level of smart grid terminal monitoring, etc., are one of the aspects of the comprehensive utilization of modern resources. 


\section{CONCLUSION}

In summary, the review of the smart grid and future challenges is a direct manifestation of China's power supply structure to meet the needs of social development. On this basis, in order to further deepen the research on the stability of smart grid, the utilization of renewable energy, demand-side design, storage equipment and the challenges and opportunities in power transmission, we analyzed the application of high-energy detection system, advanced power resource distribution system, advanced management AAML mode, and effective analysis of the external market of smart grid to provide guidance for the development of modern power transmission. Therefore, a comprehensive analysis of smart grid and future challenges will lead to a new direction for intelligent transmission planning of domestic power structure.

\section{REFERENCE}

1. Xie C.B. Discussion on the planning and design of smart grid [J]. Technology and Innovation, 2018 (01): 126-127.

2. Liu B.Y. Distribution Automation Discussion based on Smart Power Network [J]. Journal of Information, 2018 (01): 20-21.

3. Lian J.Y., Wang Y.N. Construction of Smart Grid under the Viewpoint of Internet of Things [J]. Information Record Materials, 2018 (01): 53-54.

4. Hao Z.X. Application of Intelligent Technology in Power System Automation [J]. Internal Combustion Engines and Parts, 2018 (01): 206-208.

5. Livieratos S, Vogiatzaki V E, Cottis P G. A Generic Framework for the Evaluation of the Benefits Expected from the Smart Grid[J]. Energies, 2013, 6(2):988-1008. 